\title{
Construction of an infectious clone of Zika virus stably expressing an EGFP marker in a eukaryotic expression system
}

Jing Gao ${ }^{1}$, Jiayi Chen ${ }^{1}$, Weizhi Lu' ${ }^{1}$, Jintai Cai ${ }^{1}$, Linjuan Shi ${ }^{1}$, Wei Zhao ${ }^{1,2^{*}}$ and Bao Zhang ${ }^{1,2^{*}}$

\begin{abstract}
Background: Zika virus is becoming one of the most widely transmitted arboviruses in the world. Development of antiviral inhibitor and vaccine requires an experimental system that allows rapid monitoring of the virus infection. This is achievable with a reverse genetic system. In this study, we constructed an infectious clone for Zika virus that stably expressing EGFP.

Methods: A PCR-mediated recombination approach was used to assemble the full-length Zika virus genome containing the CMV promoter, intron, EGFP, hepatitis delta virus ribozyme, and SV40 terminator sequence for cloning into the pBAC11 vector to produce recombinant PBAC-ZIKA-EGFP. ZIKA-EGFP virus was rescued by transfection of pBACZIKA-EGFP into 293T cells. The characterization of ZIKA-EGFP virus was determined by qPCR, plaque assay, CCK-8, and Western blot.
\end{abstract}

Results: Rescued ZIKA-EGFP virus exhibited stable replication for at least five generations in tissue culture. ZIKA-EGFP can effectively infect C6/36, SH-SY5Y and Vero cells, and cause cytopathic effects on SH-SY5Y and Vero cells. The inhibition of ZIKA-EGFP by NF-KB inhibitor, caffeic acid phenethyl ester was observed by fluorescence microscopy.

Conclusion: Our results suggested that Zika virus infectious clone with an EGFP marker retained it infectivity as wide-type Zika virus which could be used for drugs screening.

Keywords: Zika virus, EGFP marker, Recombinant virus, Eukaryotic expression

\section{Background}

Zika virus was transmitted mainly by the mosquito and has become epidemic worldwide. Zika virus was first discovered in the Zika Forest in Uganda in 1947, and an outbreak of Zika virus in the Americas in 2015 caused a global public health emergency [1-4]. Approximately $80 \%$ of Zika infections are asymptomatic, with the most common symptoms including fever, arthralgia, rash, myalgia, edema, vomiting, and non-purulent conjunctivitis [5, 6]. The virus can infect the placenta and blood-brain barrier

\footnotetext{
*Correspondence: zhaowei@smu.edu.cn; zhang20051005@126.com 1 Biosafety Level 3 Laboratory, School of Public Health, Southern Medical University, Guangzhou 510515, China
}

Full list of author information is available at the end of the article endothelial cells, neurons, and neural stem cells $[7,8]$ and is closely associated with diseases, such as encephalitis, fetal microcephaly, and Guillain-Barré syndrome [9-11]. Similar to other flaviviruses, the Zika genome encodes an open reading frame containing three structural proteins (C: capsid; prM: precursor of membrane; and E: envelope) that comprise the viral particles and seven genes encoding non-structural (NS) proteins (NS1, NS2A, NS2B, NS3, NS4A, NS4B, and NS5) that participate in RNA replication [12].

Recent studies show that some mutations of the Zika virus are related to host adaptation [13-16], however, the pathogenic mechanism of the virus has not been fully elucidated, and there is no available vaccine or therapeutic. The reverse genetic of Zika virus with luciferase original author(s) and the source, provide a link to the Creative Commons licence, and indicate if changes were made. The images or other third party material in this article are included in the article's Creative Commons licence, unless indicated otherwise in a credit line to the material. If material is not included in the article's Creative Commons licence and your intended use is not permitted by statutory regulation or exceeds the permitted use, you will need to obtain permission directly from the copyright holder. To view a copy of this licence, visit http://creativecommons.org/licenses/by/4.0/. The Creative Commons Public Domain Dedication waiver (http://creativeco mmons.org/publicdomain/zero/1.0/) applies to the data made available in this article, unless otherwise stated in a credit line to the data. 
or fluorescent protein have been described [1, 17, 18]. Mutso et al. reported that recombinant Zika virus carried maker genes such as nanoluciferase, enhanced green fluorescent or mCherry, which inserted between two copies of the capsid protein gene were genetically stable for at least four in vitro passages [18]. This system allows rapid monitoring of the infected cells, which provides a great convenience for the screening antiviral inhibitors.

This study describes construction of full-length infectious clone of Zika virus in a eukaryotic expression system that can be used to produce the virus along with stable expression of the enhanced green fluorescence protein (EGFP) marker. In some previous methods, recombinant Zika virus carrying EGFP marker cannot be stably passaged $[1,18]$, so it is often required to perform multiple virus rescue experiment. Virus rescue is often time-consuming and expensive, which required multiple attempts to obtain sufficient amount of rescued virus. In this study, we have established a Zika virus infectious clone with EGFP reporter which exhibited similar replication competency as wild-type ZKC2/2016 strain. Due to its inherent stability, we are able to produce high virus titer with only a single virus rescue experiment.

\section{Materials and methods}

\section{Cells, virus and reagents}

The Vero cells, $293 \mathrm{~T}$ cells, and C6/36 cells originally held in our laboratory were cultured at $37{ }^{\circ} \mathrm{C}$ with $5 \% \mathrm{CO}_{2}$ in Dulbecco's modified Eagle's medium (DMEM) supplemented with $10 \%$ fetal bovine serum (FBS; Gibco, Gaithersburg, MD, USA). SH-SY5Y cells (a thrice cloned subline of the neuroblastoma cell line SK-N-SH) obtained from KeyGEN BioTECH (NanJing, JiangSu, China) and were cultured at $37{ }^{\circ} \mathrm{C}$ with $5 \% \mathrm{CO}_{2}$ in DMEM/F12 supplemented with 10\% FBS. ZIKA-WT (ZKC2/2016, GenBank Accession NO.: KX253996), provided by the Institute of Virology of Guangdong Provincial Center for Disease Control and Prevention, was amplified in Vero cells.

Anti-Zika E and NS1 antibody were obtained from GeneTex (San Antonio, TX, USA); anti-EGFP antibodies (Abcam, Cambridge, MA, USA); DAPI, Phycoerythrin (PE) labelled goat-anti-mouse antibody, anti- $\beta$-actin, anti-mouse and anti-rabbit secondary antibodies and enhanced chemiluminescence (ECL) Horseradish Peroxidase (HRP) substrate (Bioworld, Minneapolis, MN, USA); pEGFP-N1 vector, Infusion kit, TRIzol, PrimeScript RT reagent kit, Besstar ${ }^{\mathrm{TM}}$ qPCR Master Mix, TB Green ${ }^{\mathrm{TM}}$ Premix (Takara, Dalian, China); pBAC11 (Addgene, Watertown, MA, USA); restriction enzymes $S f o \mathrm{I}$ and PacI, DH10B cells (New England Biolabs, Ipswich, MA, USA); Lipofectamine 3000 (Thermo Fisher Scientific, Waltham, MA, USA); $0.45-\mu \mathrm{m}$ membrane filter, (Pall Corporation;
Port Washington, NY, USA); polyvinylidene difluoride (PVDF) membrane (Millipore, MA,USA); bovine serum albumin (BSA), IPA lysis buffer (Genshare, Xian, China).

\section{Construction of a full-length infectious clone of Zika virus}

As shown in Fig. 1, the cytomegalovirus (CMV) promoter sequence was amplified from pEGFP-N1 vector with primers (ZCMVF: 5'-AAGGAGAAAATACCGCAT CAGGCGCGTTGACATTGATTATTGACTAG-3' and ZCMVR: $\quad 5^{\prime}$-CTGTCGCAGTCTGATTCACACAGA TCAACAACT TGT TAGCCAGAGAGCTCTGC- $3^{\prime}$, the Italics sequence is same with vector of $\mathrm{pBAC} 11$, the Underline sequence is Zika viral genomic sequence 1-31nt) synthesized by Guangzhou IGE Biotechnology Co., Ltd. (Guangzhou, China), then fused to the fragment of the Zika viral genomic sequence 1-5908nt according to the strain of ZKC2/2016 with primers (Z1F: 5'-GCA GAGCTCTCTGGCTAACAAGTTGTTGATCTGTGT GAATCAGACTGCGACAG-3' and Z1R: 5'-GGCTTT AGGCATCTCCTGGAATCTATG-3') using overlap polymerase chain reaction (PCR) method in order to generate the 5 ' half sequence of the Zika virus infectious clone (ZF). The fusion fragment of hepatitis delta virus (HDV) ribozyme sequence (688-771nt according to GenBank Accession NO.MK890235.1) with 5' additional 10707$10807 \mathrm{nt}$ of Zika genomic sequence and bovine growth hormone terminator sequence (1027-1279nt according to pCDNA3.1- vector) with $3^{\prime}$ additional $150 \mathrm{bp}$ before PacI site of pBAC11 vector were synthesized by Guangzhou IGE Biotechnology Co., Ltd., then ligated to the Zika viral sequence (5735-10807nt) using overlap PCR method with primers (Z2F: $5^{\prime}$-TTGTCTGACAAAGGC TGGAAAACGGGTCATACAG 3' and Z2R: 5' CCCCGC GGACCCCGGTTTAATTAACAGCTGGTTCTTTCC GCCTCAGAAG-3'; the Underline sequence is same with pBAC11 vector) to generate the 3 ' half sequence of the clone (ZR). Recombination reaction of three fragments of $\mathrm{ZF}, \mathrm{ZR}$ and $\mathrm{pBAC} 11$ vector previously digested with restriction enzymes (SfoI and $\mathrm{PacI}$ ) was performed using Infusion kit (Takara, Dalian). The recombinant of pBACZIKA were transformed into DH10B cells, screened with $20 \mu \mathrm{g} / \mathrm{ml}$ chloramphenicol LB agar plate and sequenced by Guangzhou IGE Biotechnology Co., Ltd. (Fig. 1b).

The intron sequence (derived from the pCI-neo vector plasmid according to GenBank Accession NO.KU870446.1) synthesized by Guangzhou IGE Biotechnology Co., Ltd. was inserted into the ZF sequence of the Zika virus (between 3128 and 3129nt) using overlap PCR method as pBAC-ZIKA mentioned to generate the sequence of the ZFi fragment (the G-to-A mutation introduced at nt3128 was used as a genetic modified marker). The ZFi and ZR fragments were recombined 

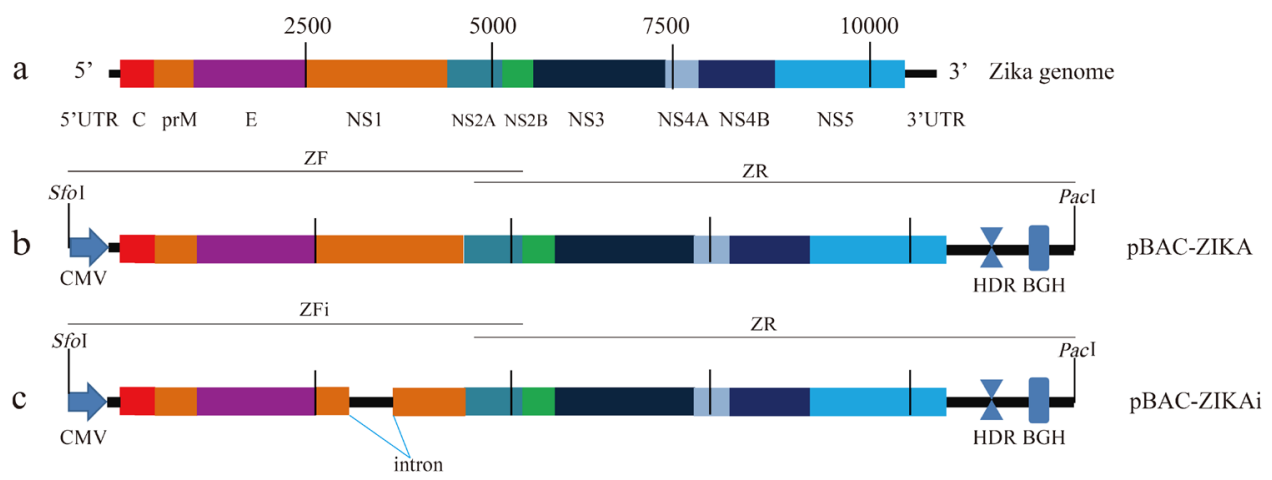

ZFE

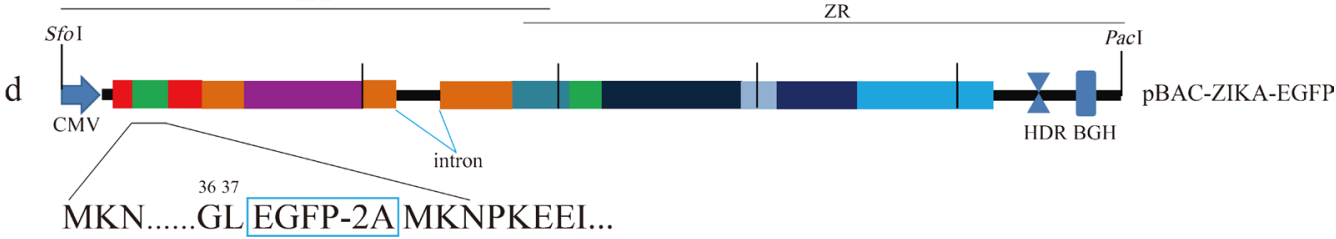

Fig. 1 a Genomic structure of the Zika virus. b Schematic diagram showing the structure of pBAC-ZIKA. CMV, HDV, and BGH denote the CMV promoter sequences, the hepatitis delta virus ribozyme sequence, and the bovine growth hormone terminator sequence, respectively. Sfol and Pacl are restriction endonucleases. PBAC-ZIKA was constructed by recombination of the ZF and ZR fragments using the restriction-digested vector. $\mathbf{c}$ Schematic diagram showing the structure of pBAC-ZIKAi. The ZFi fragment was generated by inserting the intron sequence into the genomic sequence of the Zika virus downstream of $3128 \mathrm{nt}$, followed by its recombination with ZR with the restriction-digested vector to generate pBAC-ZIKAi. d pBAC-ZIKA-EGFP was constructed by inserting the EGFP-2A gene downstream of the 37th codon of the Zika virus sequence

with pBAC11 vector digested to generate recombinant pBAC-ZIKAi (Fig. 1c).

The EGFP-2A gene (fusion sequence of EGFP gene from pEGFPN1 vector and 2A peptide of foot and mouth disease virus) was inserted downstream of the 37th codon of the Zika virus using the above-described methods to produce the fragment of ZFE with $2 \mathrm{~A}$ sequence adjoined to first codon of Zika full-length coding-region sequence. Briefly, ZFE fragment was achieved by splicing overlap extension PCR method with three PCR fragments (first was amplified from template of pBAC-ZIKAi with primers of ZCMVF and E1 5'-GCTTGAAGAGGCTGCCAG CCGGACTTCTGCTG- ${ }^{\prime}$, second was amplified from template of pEGFPN1 with primers of E2F 5'-AGTCCG GCTGGCAGCCTCTTCAAGCATGGTGACAAGGGC

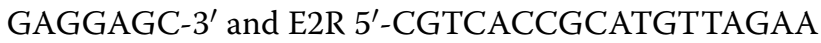
GACTTCCTCTGCCCTCCTTGTACAGCTCGTCAT GCCGA-3', third was amplified from template of pBACZIKAi with primers of E3F 5'-GAAGTCTTCTAACAT GCGGTGACGTGGAGGAGAATCCCGGCCCTATGA AAAACCCAAAAAAGAAATC-3'and Z1R. Fragments of ZFE, ZR and $\mathrm{pBAC} 11$ digested with $S f o \mathrm{I}$ and $P a c \mathrm{I}$ were recombined with Infusion kit to construct pBAC-ZIKAEGFP (Fig. 1d).

The sequences of $2 \mathrm{~A}$ peptide, intron, HDV ribozyme and $\mathrm{bGH}$ polyA signal were listed in the Additional file 1: S1.

\section{Rescue of recombinant Zika virus}

293T cells were seeded in 6-well plates and transfected with $5 \mu \mathrm{g} /$ well of the pBAC-ZIKA-EGFP or pBACZIKAi plasmid using Lipofectamine 3000 according to the manufacturer's instruction. The supernatant containing P0 generation of the ZIKA-EGFP or ZIKAi virus were harvested 7 days post transfection, passed through a $0.45-\mu \mathrm{m}$ membrane filter, and stored at $-20{ }^{\circ} \mathrm{C}$. Vero cells were infected with P0 recombinant virus at a multiplicity of infection (MOI) of 0.1. At 4 to 6 days postinfection, supernatant containing the P1 generation was collected, and Vero cells were re-infected with the P1 virus at a MOI of 1.0 in order to produce viral generations P2, P3, P4, P5.

Total RNA from Vero cells infected with ZIKA-EGFP (generation P5) was extracted using TRIzol, and EGFP expression was examined by PCR using primer $1\left(5^{\prime}\right.$ AGTTGTTGATCTGTGTGAATCAGACTG-3') targeting the Zika sequence and primer 2 (5'-CTTGTACAG CTCGTCCATGCCGAGAGTG-3') targeting the EGFP sequence. Cytopathic changes on Vero cells and expression of green fluorescence were monitored.

\section{Immune fluorescence assay}

$5 \times 10^{5}$ of Vero cells in a 6-well plate were infected with virus of ZIKA-WT and ZIKA-EGFP (generation P5) at a $\mathrm{MOI}=0.1$. At $48 \mathrm{~h}$ post-infection, cells were fixed with 
$95 \%$ ethanol for $20 \mathrm{~min}$, immune-stained with anti-E protein antibody (4G2, 1:200) for overnight at $4{ }^{\circ} \mathrm{C}$. After 5 times washing, the cells were stained with PE-labelled goat-anti-mouse antibody (1:300), then stained with DAPI. Cells were observed by fluorescent microscopy $(200 \times)$.

\section{Western blot analysis}

Western blot was used to measure levels of EGFP, NS1 and $\mathrm{E}$ proteins in Vero cells infected with ZIKA-WT, ZIKAi or ZIKA-EGFP (generation P5). Briefly, infected Vero cells in 6-well plates were harvested by addition of RIPA lysis buffer. Forty micrograms of proteins were electrophoresed in SDS-polyacrylamide gel electrophoresis gel and transferred onto a PVDF membrane. PVDF membranes were blocked with 3\% BSA buffer in tris buffered saline $/ 0.1 \%$ Tween 20 for $2 \mathrm{~h}$ at room temperature and then incubated with the following primary antibodies: anti- $\beta$-actin (1:5000); anti-Zika E protein (1:2000); anti-Zika NS1 (1:2000); anti-EGFP (1:5000) overnight at $4{ }^{\circ} \mathrm{C}$ with gentle agitation. Then the membranes were incubated with HRP-conjugated secondary antibodies for $1 \mathrm{~h}$ at room temperature with ECL reagent for exposure. Protein band were detected using a Luminescent image Analyzer (Tanon-5200, Shanghai, China).

\section{Fluorescence-based quantitative PCR (qPCR)}

Total RNA was extracted using TRIzol in accordance with the manufacturer's instructions. Thereafter, $1 \mu \mathrm{g}$ of total RNA was reverse-transcribed to cDNA using PrimeScript RT reagent kit. qPCR was performed using Bestar $^{\text {TM }}$ qPCR Master Mix with Zika E gene sense primer: 5'-CVGACATGGCTTCGGACAGY-3', antisense primer: 5'-CCCARCCTCTGTCCACYAAYG-3', and probe: 5'-AGGTGAAGCCTACCTTGACAAGCA RTCA-3' according to the following steps: preincubation $\left(95{ }^{\circ} \mathrm{C}\right.$ for $\left.120 \mathrm{~s}\right)$; 2-step amplification $\left(10 \mathrm{~s}\right.$ at $95{ }^{\circ} \mathrm{C}, 30 \mathrm{~s}$ at $60{ }^{\circ} \mathrm{C}$ for 40 cycles) with data collection at the end of $60{ }^{\circ} \mathrm{C}$; cooling $\left(37^{\circ} \mathrm{C}\right.$ for $\left.30 \mathrm{~s}\right)$. qPCR was performed using

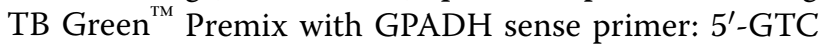
AAGGCTGAGAACGGGAA- ${ }^{\prime}$, antisense primer 5'-AAATGAGCCCCAGCCTTCTC-3' according to the following steps: preincubation $\left(95{ }^{\circ} \mathrm{C}\right.$ for $\left.30 \mathrm{~s}\right)$; 3-step amplification $\left(15 \mathrm{~s}\right.$ at $95^{\circ} \mathrm{C}, 15 \mathrm{~s}$ at $55^{\circ} \mathrm{C}, 15 \mathrm{~s}$ at $72{ }^{\circ} \mathrm{C}$ for 40 cycles) with data collection at the end of $72{ }^{\circ} \mathrm{C}$; melting $\left(95{ }^{\circ} \mathrm{C}\right.$ for $10 \mathrm{~s}, 65^{\circ} \mathrm{C}$ for $30 \mathrm{~s}, 97^{\circ} \mathrm{C}$ for $1 \mathrm{~s}$ ); cooling $\left(37^{\circ} \mathrm{C}\right.$ for $30 \mathrm{~s}$ ). Zika viral mRNA expression levels were normalized to those of GAPDH using the $2^{-\Delta \Delta \mathrm{Ct}}$ method. The results are expressed as expression fold changes. qPCR can detect more than 1copy/ $\mu \mathrm{L}$ DNA determined by standard curve with plasmid.

\section{Viral replication ability}

Growth kinetics of ZIKA-EGFP was compared with ZIKA-WT in vitro. Briefly, Vero cells were seed in 12 -well plates $\left(2 \times 10^{5}\right.$ cells/well $) .12 \mathrm{~h}$ later, both viruses were inoculated into individual wells $(\mathrm{MOI}=0.1)$. Supernatants and cells were collected at $12 \mathrm{~h}, 24 \mathrm{~h}$, and $48 \mathrm{~h}$ post-infection, and total RNA was extracted and reverse transcribed. The replication of viral RNA in cells was measured using qPCR [19]. An assay to determine the $50 \%$ tissue culture infectious dose $\left(\operatorname{TCID}_{50}\right)$ with Vero cells was used to determine viral titer in the supernatant, with TCID $_{50}$ values calculated according to the Reed-Muench method.

\section{Viral toxic effects on cells}

ZIKA-WT and ZIKA-EGFP were used to infect SHSY5Y cells at a MOI of 1.0. At $48 \mathrm{~h}$ and $72 \mathrm{~h}$ postinfection, cell viabilities were measured using a Cell Counting Kit-8 (CCK-8; Dojindo, Shanghai, China) assay. Cytopathic effects were observed using a microscope (Eclipse E200, Nikon, Tokyo, Japan).

Plaque assay was used to determine the cytopathic effect of ZIKA-WT and ZIKA-EGFP. Vero cells were seeded in 24-well plates at $5 \times 10^{4}$ cells/well, and the following day, an appropriate viral titer was used to infect the cells. At 2-h post-infection, 2\% carboxymethylcellulose was used to overlay the cells, and after 6 days, Crystal Violet staining was performed to observe plaque sizes.

\section{Infectivity to the $\mathrm{C} 6 / 36$ cells}

$5 \times 10^{4} \mathrm{C} 6 / 36$ cells/well were seeded in 24-well plates. After $24 \mathrm{~h}$, cells were infected by ZIKA-EGFP with MOI $=0.1$. At $48 \mathrm{~h}, 72 \mathrm{~h}$ and $144 \mathrm{~h}$ post-infection, cells were observed by fluorescent microscope (Eclipse E200, Nikon, Japan).

\section{Inhibitory effect of caffeic acid phenethyl ester (CAPE; Selleck, Shanghai, China) on ZIKA-EGFP Viral Replication} Vero cells were infected with ZIKA-EGFP and ZIKAWT $(\mathrm{MOI}=0.1)$ and cultured with $2 \mathrm{ml}$ media containing $10 \mu \mathrm{M}$ CAPE for $48 \mathrm{~h}$. Relative expression levels of the viruses were measured using qPCR. Additionally, fluorescence intensity was observed under a fluorescence microscope.

\section{Statistics analysis}

All experiments were repeated at least three times. Data were presented as mean \pm SEM. Results were analyzed using Graphpad Prism (GraphPad Software Inc., La Jolla, CA). Comparison between experimental groups was made by Student's t-test and one-way 
ANOVA. A value of $P<0.05$ was considered statistically significant. $\left({ }^{*} P<0.05,{ }^{* *} P<0.01,{ }^{* * * *} P<0.001,{ }^{\text {n.s }} P>0.05\right)$.

\section{Results}

\section{Production of rescued virus}

The pBAC-ZIKA-EGFP clone generated as described in Fig. 1 was used to transfect 293T cells, supernatant was collected at 7-days post-transfection and used to infect Vero cells. The viruses were passaged every 4-6 days. Total RNA was extracted from the cells after infection and identified EGFP expression by PCR, demonstrating the stability of the ZIKA-EGFP viral sequence following passaging (Fig. 2a). At 48-h post-infection with ZIKAEGFP (generation P5), green fluorescence was clearly visible under fluorescence microscope (Fig. 2b), at the same time, some cells could be observed rounded, crinkled, and even shed in the bright field of microscopy, which suggested that recombinant ZIKA-EGFP could induce cytopathic effect in Vero cells (Fig. 2b). We used Western blot experiment to confirm that ZIKA-EGFP (generation P5) stably express EGFP using NS1 and E proteins of Zika virus as references in Vero cells at $48 \mathrm{~h}$ post-infection. a

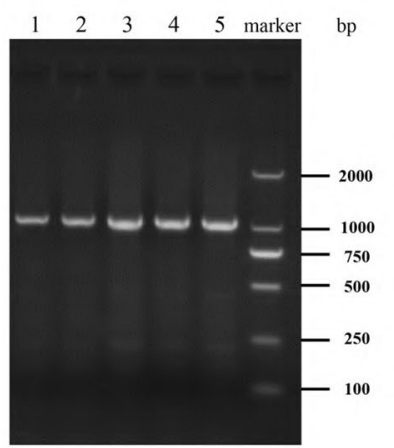

b

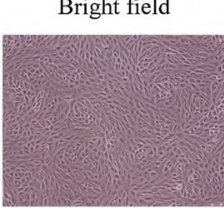

no virus

ZIKA-EGEP

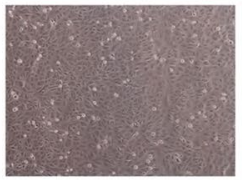

Fluorescence

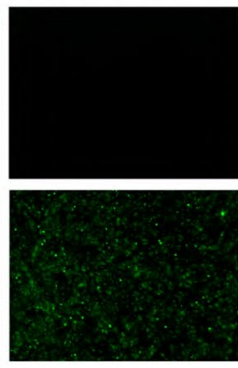

c

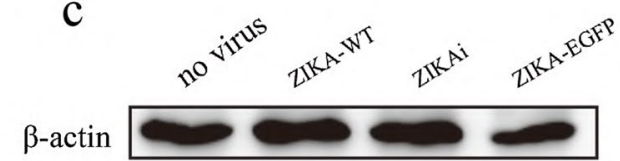

Envelope

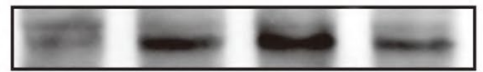

NS1

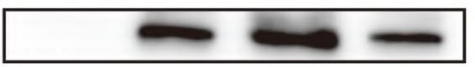

EGFP

1. ZIKA-EGFP P1

2. ZIKA-EGFP P2

3. ZIKA-EGFP P3

4. ZIKA-EGFP P4

5. ZIKA-EGFP P5

d

Time post infection

48 (hour)

Bright field
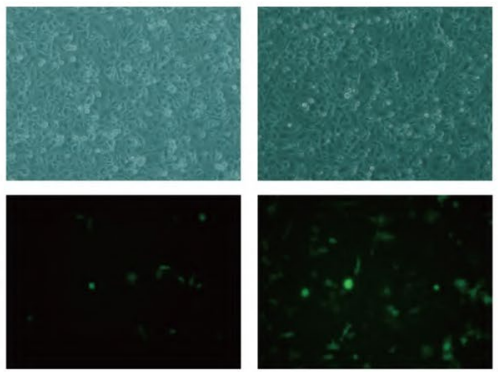

144

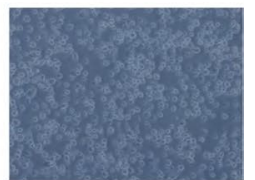

Fluorescence

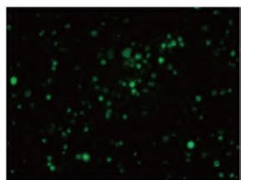

e

DAPI

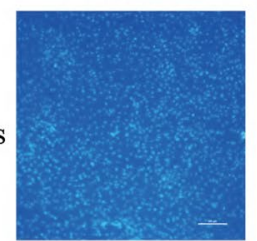

ZIKA-WT

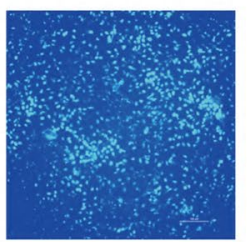

ZIKA-EGFP

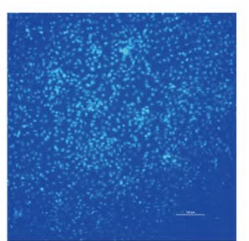

EGFP
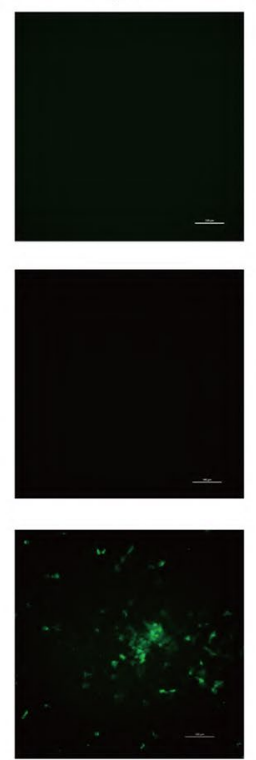

E protein
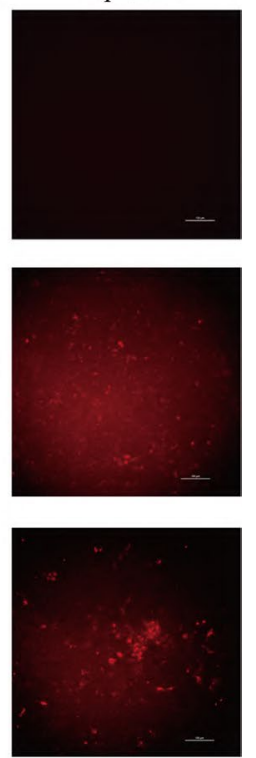

Fig. 2 Rescue of recombinant ZIKA-EGFP. a Detection of EGFP by electrophoresis. (1-5 lanes are generation p1-p5 of recombinant ZIKA-EGFP, respectively) $\mathbf{b}$ Fluorescence of Vero cells infected with the ZIKA-EGFP (generation P5) for $48 \mathrm{~h}(\times 200)$. c Western blot detection of E gene, NS1 gene, and EGFP gene levels in Vero cells infected with the ZIKA-WT, ZIKAi (generation P5), and ZIKA-EGFP (generation P5) for $48 \mathrm{~h}$. Western blot analysis was repeated three times and representative result was exhibited. $\mathbf{d}$ Fluorescence of C6/36 cells infected with ZIKA-EGFP at different time points. e. The localization of EGFP and E protein for ZIKA-EGFP in Vero cells ( $\times 200)$ 
The rescued ZIKAi virus (generation P5) expressed NS1 and $E$ proteins, which indicated that rescued ZIKAi virus by eukaryotic expression system was steady (Fig. 2c). Zika virus is transmitted by Aedes mosquitoes. Therefore, we want to know if ZIKA-EGFP can successfully infect $\mathrm{C} 6 / 36$ cells. We can observe more cells express EGFP over time when $\mathrm{C6} / 36$ cells were infected with ZIKA-EGFP, suggesting that ZIKA-EGFP can also infect mosquito cell line and replicate in cells (Fig. 2d). Moreover, ZIKA-EGFP could be passaged at least six generations with EGFP expression (data not shown). To further confirmed that the genome ZIKA-EGFP stability, EGFP and $\mathrm{E}$ protein expression were observed by immune fluorescence (Fig. 2e) which showed that EGFP and E protein had colocalized in the ZIKA-EGFP group.

\section{Viral replication ability}

Levels of viral RNA in ZIKV-EGFP infected Vero cells $(\mathrm{MOI}=0.1)$ at $12 \mathrm{~h}, 24 \mathrm{~h}$, and $48 \mathrm{~h}$ post-infection were measured using qPCR. The results showed that ZIKAEGFP viral RNA could be amplified in Vero cells and was comparable with ZIKA-WT (Fig. 3a), which revealed that no significant difference in the replication kinetic between ZIKA-EGFP and ZIKA-WT. Culture supernatants were then collected and subjected to $\mathrm{TCID}_{50}$ assay. Our data showed similar titers between ZIKA-EGFP and ZIKA-WT (Fig. 3b), indicating similar viral replication kinetic between ZIKA-EGFP and ZIKA-WT.

\section{Viral cytopathic effects on cells}

Neurotropism of Zika virus is associated with encephalitis, fetal microcephaly, and Guillain-Barré syndrome closely. We include SH-SY5Y cell line, a subline of neuroblastoma, to study the replication competency of ZIKAEGFP. After infection of SH-SY5Y cells with ZIKA-EGFP and ZIKA-WT (MOI=1.0) for $48 \mathrm{~h}$, we observed prominent cytopathic effects (data not shown). Additionally, CCK-8 assays showed that cells viability of ZIKA-EGFPinfected cells decreased significantly in time-dependent manner, but ZIKA-EGFP cytopathic effect was weaker than ZIKA-WT, with cells viability of $75 \%$ and $49 \%$ for ZIKA-EGFP and ZIKA-WT at $72 \mathrm{~h}$ post-infection, respectively (Fig. 4a). Plaque assay results showed that ZIKA-EGFP did not result in plaque formation in Vero cells, while ZIKA-WT led to the formation of prominent, perfectly round, and uniform plaques after 6 days postinfection (Fig. 4b). Green fluorescence cell clusters were observed indicating that ZIKA-EGFP did infect Vero cells (Fig. 4c).

\section{Inhibitory effect of CAPE on viral replication}

Vero cells infected with ZIKA-EGFP and ZIKA-WT $(\mathrm{MOI}=0.1)$ were treated with $10 \mu \mathrm{M}$ CAPE for $48 \mathrm{~h}$. The qPCR results revealing that CAPE significantly inhibited viral replication (Fig. 5a). These findings were further confirmed by immunofluorescence assay (Fig. 5b).

\section{Discussion}

The reverse genetics approach is an important method to study viral gene function and virulence, and as an important tool to screen for anti-Zika virus therapeutic. The presence of cryptic bacterial promoters in flaviviruses has increased the challenges associated with construction of infectious clones $[20,21]$. The common solution to this issue is mutation of the cryptic promoter [22] or utilization of a single-copy plasmid-based bacterial artificial chromosome [20]. In this study, we used a bacterial artificial chromosome to reduce the toxicity of the viral DNA in order to obtain a full-length clone of Zika virus.
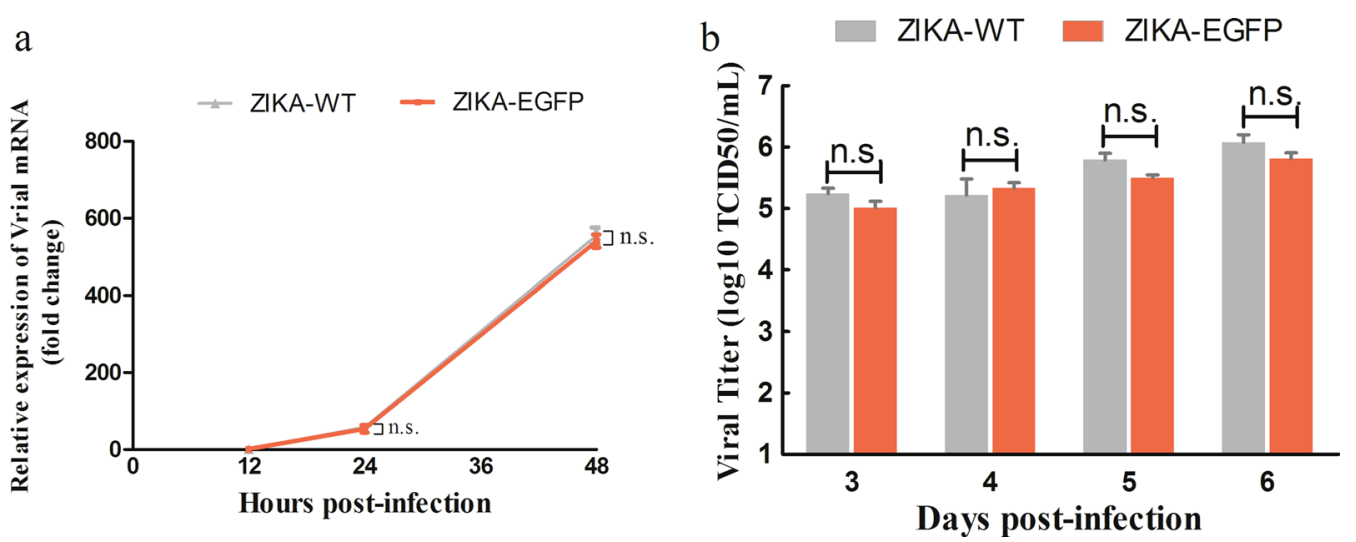

Fig. 3 Viral kinetics of ZIKA-WT and ZIKA-EGFP in Vero cells. Vero cells were infected with ZIKA-WT and ZIKA-EGFP, and a intracellular viral RNA expression was detected at various time points by qPCR. b Viral titer in culture supernatant was determined by TCID 50 assay. All experiments were repeated three times and each group contained three replicates 
a

SH-SY5Y cells (MOI 1.0)

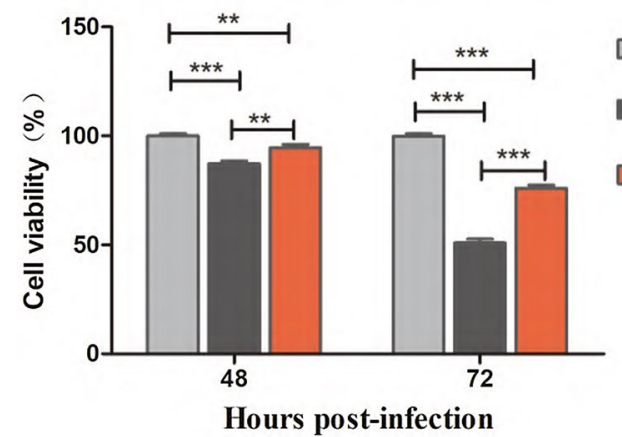

C

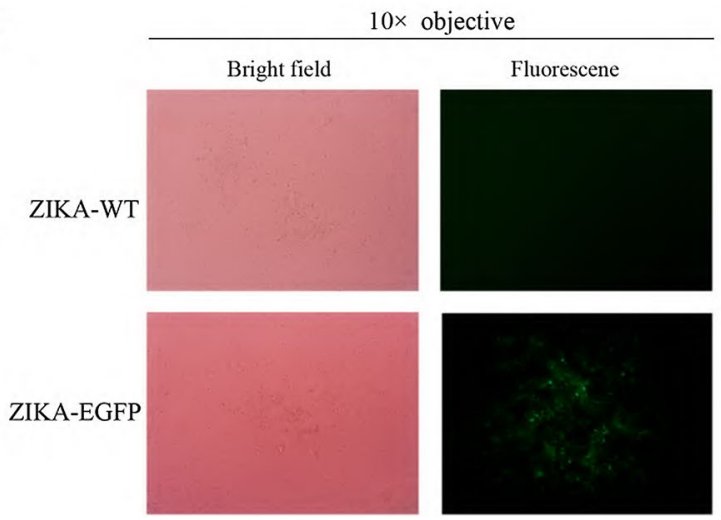

CON

ZIKA-WT

ZIKA-EGFP

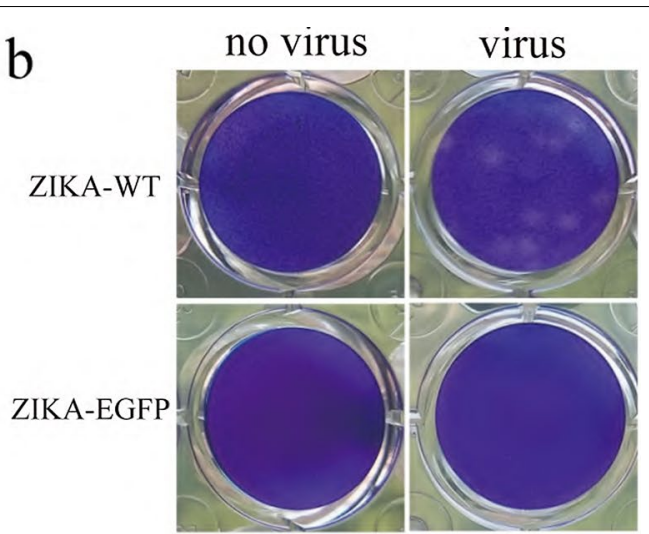

$20 \times$ objective
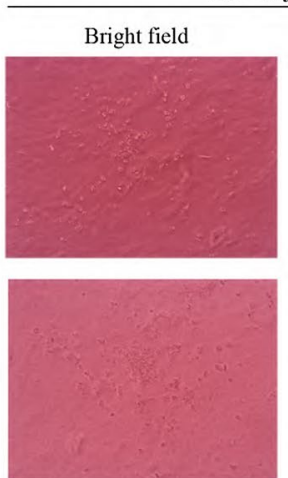
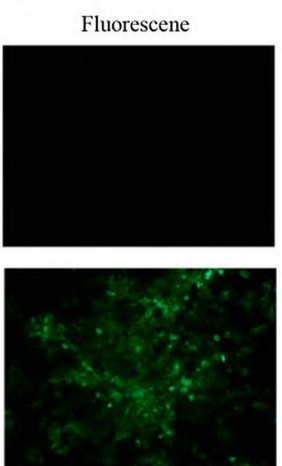

Fig. 4 Comparison of ZIKA-EGFP and ZIKA-WT toxic effects cells. a SH-SY5Y cells were incubated with ZIKA-WT and ZIKA-EGFP (MOI=1.0), and cell viability was determined by CCK-8 assay at 48- and 72-h post-infection. b Cytopathic effect was compared by plaque assays with Vero cells. c Fluorescent cells aggregated due to the cytopathic effects associated with ZIKA-EGFP in Vero cells. CCK-8 tests were repeated three times and each group contained six replicates. ${ }^{* *} P<0.01,{ }^{* * *} P<0.001$
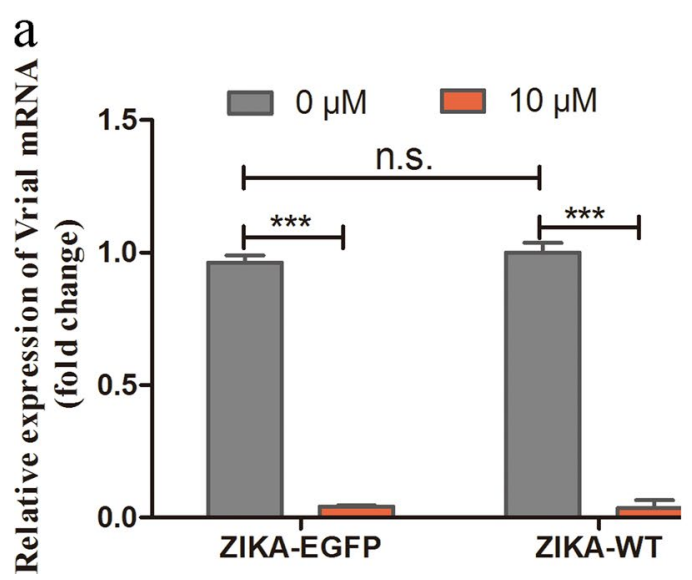

b CAPE $(\mu \mathrm{M})$

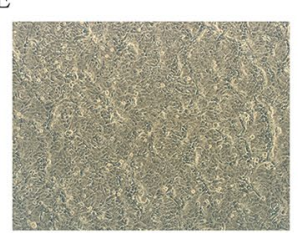
Fluorescence

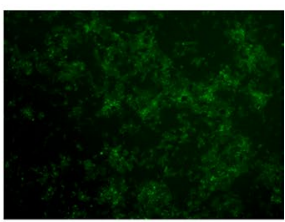

10
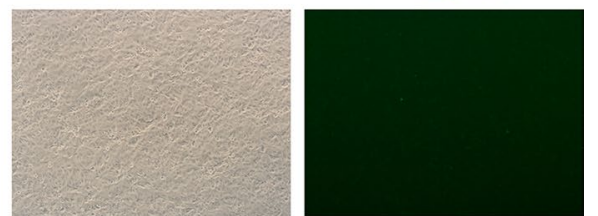

Fig. 5 CAPE inhibition of ZIKA-EGFP infection. a Vero cells infected with ZIKA-WT and ZIKA-EGFP at a MOI of 0.1 were cultured in the presence and absence of $10 \mu \mathrm{M}$ CAPE for $48 \mathrm{~h}$, followed by detection of Zika viral E gene expression by qPCR. $\mathbf{b}$ Fluorescent cells were observed under a fluorescence microscope $(\times 200)$. The qPCR assays were repeated three times and each group contained three replicates. ${ }^{* * *} P<0.001$, n.S., not significant 
Infectious clones can be constructed in three types of systems. The first is the in vitro transcription system [2325], where a full-length DNA clone is used as a template to transcribe viral mRNA molecules using T7 or SP6 RNA polymerase. The purified viral RNA is then used to transfect cells via electrotransfection or other methods to produce rescued viruses. This method requires highly pure mRNA and often expensive and time-consuming. The second approach involves use of eukaryotic systems $[1,25,26]$, where a clone containing the full-length viral sequence is constructed and placed under the control of a eukaryotic promoter, such as CMV or SV40. Upon transfection of target cells with the plasmid, viral mRNA can be generated using the transcription machinery of the eukaryotic cells, enabling subsequent rescue of the viruses produced in the cells. This method generally does not require expensive reagents or instruments, is simple to perform, and the conditions are easily optimizable. However, the limitation of this method involves the need for transcription inside the nucleus during generation of viral mRNA. We failed to produce a rescued Zika virus because there was a fragment deletion of mRNA, which is probably related to abnormal RNA splicing. We then inserted an intron sequence to guide correct RNA splicing at 3128nt of Zika virus genome sequence which successfully to rescue virus. Avila-Perez et al. and Chen et al. used this method to construct infectious clones and did not observe incorrect pre-mRNA splicing, which could be due to sequence differences in different viral strain $[20,22]$. The third approach is using recombination systems with overlapping fragments $[27,28]$. In this system, the full-length DNA clone and the transcription-control elements are amplified as three fragments, which are then transfected into target cells via electrotransfection. The complete viral genome sequence is then generated in the cells by homologous recombination to subsequently produce the rescued viruses. This method can avoid the toxic effect of promoter of the cryptic bacterial, however, the transfection conditions need to be optimized, and the efficiency of homologous recombination can greatly affect viral production.

To rapidly screen drugs for inhibiting the viral replication and study the mechanism of replication and translation, Xie et al. constructed replication system that the chimeric replicon of Rluc gene and viral genome could stably passage, but not produce virus particles [29].

To facilitate investigations of Zika virus pathogenesis, previous studies attempted to include a marker, such as EGFP or luciferase at C25 [1], C33 [17], or other positions, in the Zika viral genome. However, most attempts did not lead to stable expression of the markers. Gadea et al.found that the recombinant ZIKV-GFP with EGFP gene at the downstream of the first 33 amino acids of
Capsid protein (C33) was unstable and the virus would lose EGFP expression after 3 generation passages. Zou et al. found recombinant Luc-DENV-2 with RLuc gene inserted at first 38 amino acids of Capsid protein (C38) was stable for at least 5 generations [30]. Upon analysis of the secondary structure of the viral RNA genome of Zika virus and Dengue-2 virus, we found that Zika virus has similar structure with Dengue- 2 on the $5^{\prime}$ and $3^{\prime}$ terminal of genomic sequence. These evidences indicated that Zika virus genome might exist sites for exogenous gene insertion which could stably passage. Dengue virus C38 (Leucine amino acid) locates in the middle of the sixth stem-loop structure. We designed the marker-insertion site at the C37 position (Leucine amino acid), which corresponds to the site of C38 of Dengue- 2 virus. We proved that expression of EGFP is stable during passages in multiple dimensions including genome level, protein level and direct observation of green fluorescence. Our results showed that insertion of EGFP at the C37 position led to a ZIKA-EGFP viral sequence that remained stable in the genome for at least five generations or more, with no adverse effect on the range of cells that could be infected (the ZIKA-EGFP was able to infect Vero, SH-SY5Y, and C6/36). Additionally, we found that the viral-replication kinetic does not differ significantly from ZIKA-WT, determined by qPCR. Also, no significant difference in viral titer between ZIKA-EGFP and ZIKAWT $(p=0.113)$ at $72 \mathrm{~h}$ post-infection. Moreover, ZIKAEGFP was cytopathogenic and led to cytopathic effects, determined with CCK-8 test but weaker than ZIKAWT. ZIKA-EGFP does not form plaque in Vero cells, but apparent cell clumps were observed under fluorescent microscopy, that consisted with the report, the mechanism is not clear. These findings indicated the effectiveness of constructing a full-length infectious clone of ZIKA-EGFP in a eukaryotic system. Furthermore, we carried out (1) that EGFP insertion at positions C36 and C38 also produced stable expression clones; (2) that the vector constructed using this method contained two cyclization sequences (CS) sites. Introduction of a mutation into the second CS site resulted in no change in viral titer; (3) that insertion of Renilla luciferase at position C37 resulted in stable expression for at least three generations (data not shown). Unfortunately, we could not demonstrate the stability of recombinant virus containing EGFP in vivo.

To validate the use of ZIKA-EGFP for monitoring drug effectiveness, we performed an experiment using CAPE, which was shown to inhibit Zika viral replication in our previous experiments. The mechanism of CAPE's inhibition effect on Zika virus replication which we are working on is not clear. We used CAPE only as a model to prove the ZIKA-EGFP to be effective for drug study. The 
results showed that treatment with the drug significantly suppressed EGFP expression, which was consistent with results observed following inhibition of RNA replication. These findings suggested that ZIKA-EGFP could be applicable for use in drug screening.

\section{Conclusions}

In summary, we successfully constructed an infectious clone of the full-length genome of Zika virus stably expressing an EGFP reporter in a eukaryotic expression system. This system can be used to study gene function and for antiviral drug screening.

\begin{abstract}
Abbreviations
NS: Non-structural; E: Envelop; EGFP: Green fluorescence protein; DMEM: Dulbecco's modified Eagle's medium; FBS: Fetal bovine serum; PE: Phycoerythrin; ECL: Enhanced chemiluminescence; HRP: Horseradish Peroxidase; PVDF: Polyvinylidene difluoride; BSA: Bovine serum albumin; MOI: Multiplicity of infection; CMV: Cytomegalovirus; HDV: Hepatitis delta virus; GPCR: Fluorescence-based quantitative PCR; TCID50: 50\% Tissue culture infectious dose; CCK-8: Cell Counting Kit-8; CAPE: Caffeic Acid Phenethyl Ester; CS: Cyclization sequences.
\end{abstract}

\section{Supplementary Information}

The online version contains supplementary material available at https://doi. org/10.1186/s12985-021-01622-z.

Additional file 1: S1 Sequences of 2A peptide, intron, etc.

\section{Acknowledgements}

We are grateful for Institute of Virology of Guangdong Provincial Center for Disease Control and Prevention for kindly providing the wide type Zika virus.

\section{Authors' contributions}

JG and BZ designed the experiments. JG, JYC, JTC and LS carried out the experiments. JYC and JG analyzed the data. JG wrote the paper. BZ and WZ approved the final version of the paper. All authors read and approved the final manuscript

\section{Funding}

This work was supported by the National Natural Science Foundation (Nos. 31470271, 81730110, and 31670168), Guangdong Provincial Science and Technology (Nos. 2018B020207006 and 201803040006) and Open Project of Guangdong Provincial Key Laboratory of Tropical Disease Research (No. KLTDR202005). The funds were basically provided to support the education of the first author, and the laboratory and field expenses. The funding bodies do not have any role in the design of the study and collection, analysis, and interpretation of data and in writing the manuscript.

\section{Availability of data and materials}

Not applicable.

\section{Declarations}

Ethics approval and consent to participate

Not applicable.

\section{Consent for publication}

Not applicable.

\section{Competing interests}

The authors declare that they have no competing interests.

\section{Author details}

${ }^{1}$ Biosafety Level 3 Laboratory, School of Public Health, Southern Medical University, Guangzhou 510515, China. ${ }^{2}$ Guangdong Provincial Key Laboratory of Tropical Disease Research, School of Public Health, Southern Medical University, Guangzhou 510515, China.

Received: 21 September 2020 Accepted: 14 July 2021

Published online: 19 July 2021

\section{References}

1. Shan C, Xie X, Muruato AE, Rossi SL, Roundy CM, Azar SR, et al. An infectious cDNA clone of Zika virus to study viral virulence, mosquito transmission, and antiviral inhibitors. Cell Host Microbe. 2016;19(6):891-900.

2. Dick GW, Kitchen SF, Haddow AJ. Zika virus. I. Isolations and serological specificity. Trans R Soc Trop Med Hyg. 1952;46(5):509-20.

3. Dick GW. Zika virus. II. Pathogenicity and physical properties. Trans R Soc Trop Med Hyg. 1952;46(5):521-34.

4. Organization WH. Zika virus outbreaks in the Americas. Wkly Epidemiol Rec. 2015;90(45):609-10.

5. Lanciotti RS, Kosoy OL, Laven JJ, Velez JO, Lambert AJ, Johnson AJ, et al. Genetic and serologic properties of Zika virus associated with an epidemic, Yap State, Micronesia, 2007. Emerg Infect Dis. 2008;14(8):1232-9.

6. Mladinich MC, Schwedes J, Mackow ER. Zika virus persistently infects and is basolaterally released from primary human brain microvascular endothelial cells. MBio. 2017;8(4):e00952-e1017.

7. Himmelsbach $K$, Hildt E. Identification of various cell culture models for the study of Zika virus. World J Virol. 2018;7(1):10-20.

8. Rolfe AJ, Bosco DB, Wang J, Nowakowski RS, Fan J, Ren Y. Bioinformatic analysis reveals the expression of unique transcriptomic signatures in Zika virus infected human neural stem cells. Cell Biosci. 2016;6(1):42.

9. Mlakar J, Korva M, Tul N, Popovic M, Poljsak-Prijatelj M, Mraz J, et al. Zika virus associated with microcephaly. N Engl J Med. 2016;374(10):951-8.

10. De Carvalho NS, De Carvalho BF, Fugaca CA, Doris B, Biscaia ES. Zika virus infection during pregnancy and microcephaly occurrence: a review of literature and Brazilian data. Braz J Infect Dis. 2016;20(3):282-9.

11. Calvet G, Aguiar RS, Melo ASO, Sampaio SA, de Filippis I, Fabri A, et al. Detection and sequencing of Zika virus from amniotic fluid of fetuses with microcephaly in Brazil: a case study. Lancet Infect Dis. 2016;16(6):653-60.

12. Kuno G, Chang GJ. Full-length sequencing and genomic characterization of Bagaza, Kedougou, and Zika viruses. Arch Virol. 2007;152(4):687-96.

13. Duggal NK, McDonald EM, Weger-Lucarelli J, Hawks SA, Ritter JM, Romo $\mathrm{H}$, et al. Mutations present in a low-passage Zika virus isolate result in attenuated pathogenesis in mice. Virol J. 2019;530:19-26.

14. Rossi SL, Ebel GD, Shan C, Shi PY, Vasilakis N. Did Zika virus mutate to cause severe outbreaks? Trends Microbiol. 2018;26(10):877-85.

15. Lin D, Li L, Xie T, Yin Q, Saksena N, Wu R, et al. Codon usage variation of Zika virus: the potential roles of NS2B and NS4A in its global pandemic. Virus Res. 2018;247:71-83.

16. Xia H, Luo H, Shan C, Muruato AE, Nunes BTD, Medeiros DBA, et al. An evolutionary NS1 mutation enhances Zika virus evasion of host interferon induction. Nat Commun. 2018;9(1):414.

17. Gadea G, Bos S, Krejbich-Trotot P, Clain E, Viranaicken W, El-Kalamouni C, et al. A robust method for the rapid generation of recombinant Zika virus expressing the GFP reporter gene. Virol. 2016;497:157-62.

18. Mutso M, Saul S, Rausalu K, Susova O, Žusinaite E, Mahalingam S, et al. Reverse genetic system, genetically stable reporter viruses and packaged subgenomic replicon based on a Brazilian Zika virus isolate. J Gen Virol. 2017;98(11):2712-24.

19. Yu J, Liu X, Ke C, Wu Q, Lu W, Qin Z, et al. Effective suckling C57BL/6, Kunming, and BALB/C mouse models with remarkable neurological manifestation for Zika virus infection. Viruses. 2017;9(7):165.

20. Avila-Perez G, Nogales A, Martin V, Almazan F, Martinez-Sobrido L. Reverse genetic approaches for the generation of recombinant Zika virus. Viruses. 2018;10(11):597.

21. Avila-Perez G, Park JG, Nogales A, Almazan F, Martinez-Sobrido L. Rescue of recombinant Zika virus from a bacterial artificial chromosome cDNA clone. J Vis Exp. 2019;(148):e59537. 
22. Chen Y, Liu T, Zhang Z, Chen M, Rong L, Ma L, et al. Novel genetically stable infectious clone for a Zika virus clinical isolate and identification of RNA elements essential for virus production. Virus Res. 2018;257:14-24.

23. Zhao F, Xu Y, Lavillette D, Zhong J, Zou G, Long G. Negligible contribution of M2634V substitution to ZIKV pathogenesis in AG6 mice revealed by a bacterial promoter activity reduced infectious clone. Sci Rep. 2018;8(1):10491.

24. Marquez-Jurado S, Nogales A, Avila-Perez G, Iborra FJ, Martinez-Sobrido L, Almazan F. An alanine-to-valine substitution in the residue 175 of Zika virus NS2A protein affects viral RNA synthesis and attenuates the virus in vivo. Viruses. 2018;10(10):547.

25. Liu ZY, Yu JY, Huang XY, Fan H, Li XF, Deng YQ, et al. Characterization of cis-acting RNA elements of Zika virus by using a self-splicing ribozymedependent infectious clone. J Virol. 2017;91(21):e00484-e517.

26. Schwarz MC, Sourisseau M, Espino MM, Gray ES, Chambers MT, Tortorella D, et al. Rescue of the 1947 Zika virus prototype strain with a cytomegalovirus promoter-driven cDNA clone. mSphere. 2016;1(5):e00246-e316.

27. Usme-Ciro JA, Lopera JA, Enjuanes L, Almazan F, Gallego-Gomez JC. Development of a novel DNA-launched dengue virus type 2 infectious clone assembled in a bacterial artificial chromosome Virus Res. 2014;180:12-22.

28. Tsetsarkin KA, Kenney H, Chen R, Liu G, Manukyan H, Whitehead SS, et al. A full-length infectious CDNA clone of Zika virus from the 2015 epidemic in Brazil as a genetic platform for studies of virus-host interactions and vaccine development. MBio. 2016;7(4):e01114-e1116.

29. Xie X, Zou J, Shan C, Yang Y, Kum DB, Dallmeier K, et al. Zika virus replicons for drug discovery. EBioMedicine. 2016;12:156-60.

30. Zou G, Xu HY, Qing M, Wang OY, Shi PY. Development and characterization of a stable luciferase dengue virus for high-throughput screening. Antiviral Res. 2011;91(1):11-9.

\section{Publisher's Note}

Springer Nature remains neutral with regard to jurisdictional claims in published maps and institutional affiliations.
Ready to submit your research? Choose BMC and benefit from:

- fast, convenient online submission

- thorough peer review by experienced researchers in your field

- rapid publication on acceptance

- support for research data, including large and complex data types

- gold Open Access which fosters wider collaboration and increased citations

- maximum visibility for your research: over $100 \mathrm{M}$ website views per year

At BMC, research is always in progress.

Learn more biomedcentral.com/submissions 